\title{
Tracheobronchitis with stridor in a patient with ulcerative colitis
}

\author{
Zahava C. Farkas ${ }^{1}$, Sevak Keshishyan ${ }^{2}$, Raja Chandra Chakinala ${ }^{1}$, Shalom Frager ${ }^{3}$, Faisal Saeed ${ }^{4}$, Yasmin \\ Yusuf $^{5}$, Chaitanya Shilagani ${ }^{6}$, Roxana Bodin ${ }^{3}$, Kassem Harris ${ }^{2}$, Wilbert S. Aronow ${ }^{2,4}$ \\ ${ }^{1}$ Department of Medicine, ${ }^{2}$ Division of Pulmonary and Critical Care, ${ }^{3}$ Division of Gastroenterology and Hepatobiliary Diseases, ${ }^{4}$ Division of \\ Cardiology, ${ }^{5}$ Department of Pathology, ${ }^{6}$ Department of Radiology, Westchester Medical Center and New York Medical College, Valhalla, NY, USA \\ Correspondence to: Wilbert S. Aronow, MD, FACC, FAHA. Professor of Medicine, Division of Cardiology, Westchester Medical Center and New York \\ Medical College, Macy Pavilion, Room 141, Valhalla, NY 10595, USA. Email: WSAronow@aol.com.
}

\begin{abstract}
Bronchopulmonary involvement is a rare but well documented extraintestinal manifestation of inflammatory bowel disease (IBD). IBD-related pulmonary disease can range from subglottic stenosis to tracheobronchitis to interstitial lung disease and is often misdiagnosed on initial presentation. We present a case of tracheobronchitis with stridor in a 23-year-old-woman with well controlled ulcerative colitis (UC).
\end{abstract}

Keywords: Tracheobronchitis; ulcerative colitis (UC); inflammatory bowel disease (IBD)

Submitted Jul 05, 2018. Accepted for publication Oct 19, 2018.

doi: $10.21037 /$ atm.2018.10.47

View this article at: http://dx.doi.org/10.21037/atm.2018.10.47

\section{Introduction}

Extraintestinal manifestations of inflammatory bowel disease (IBD) develop in $21 \%$ to $41 \%$ of patients with a higher incidence in those with Crohn's disease (CD) as compared to ulcerative colitis (UC) $(1,2)$. Bronchopulmonary involvement is rare, occurring in only $0.21 \%$ to $0.4 \%$ of patients with IBD. Any part of the respiratory system can be involved. Bronchiectasis is the most common reported finding, however, tracheobronchitis, pleural and parenchymal disease can also occur $(3,4)$. The rare nature of this finding and lack of physician awareness is perhaps why it is often misdiagnosed as bronchial asthma on initial presentation (5). We present a case of UC-related tracheobronchitis in a patient who presented with stridor to emphasize the importance of maintaining a high clinical suspicion for pulmonary manifestations of IBD.

\section{Case presentation}

A 23-year-old woman presented to an outside hospital in acute respiratory distress with audible stridor. Three weeks prior, she had presented to that same hospital with shortness of breath, and was treated with levofloxacin for presumed community acquired pneumonia. She developed progressively worsening dyspnea, later associated with intermittent fever, night sweats, sore throat, voice hoarseness, productive cough and an unintentional ninepound weight, prompting a return to the emergency department. This time she was noted to be tachypneic, in respiratory distress and with audible stridor.

Stridor in adults is commonly caused by an abscess, tumor, laryngomalacia, subglottic stenosis or upper airway swelling, such as anaphylaxis, tracheitis or respiratory papillomatosis. It can be a sign of a life-threatening emergency and treatment should not be delayed. In this case, the patient received intravenous dexamethasone, racemic epinephrine, helium-oxygen inhalation therapy and empiric broad-spectrum antibiotic coverage. Computed tomography of the neck and thorax showed diffuse circumferential nodular tracheal and central bronchial thickening (Figure 1), and nodules within the left upper lobe, thought to be secondary to infection versus inflammation in the setting of tracheobronchitis. Two days later she was transferred to the medical intensive care unit at our institution for ENT evaluation; by then, the previously noted stridor had resolved and she never required intubation.

Of note, this patient was a non-smoker with no history of pulmonary disease, sick contacts or known tuberculosis exposures. She emigrated from the Dominican Republic in 2009 but had no recent travel. Past medical history was 

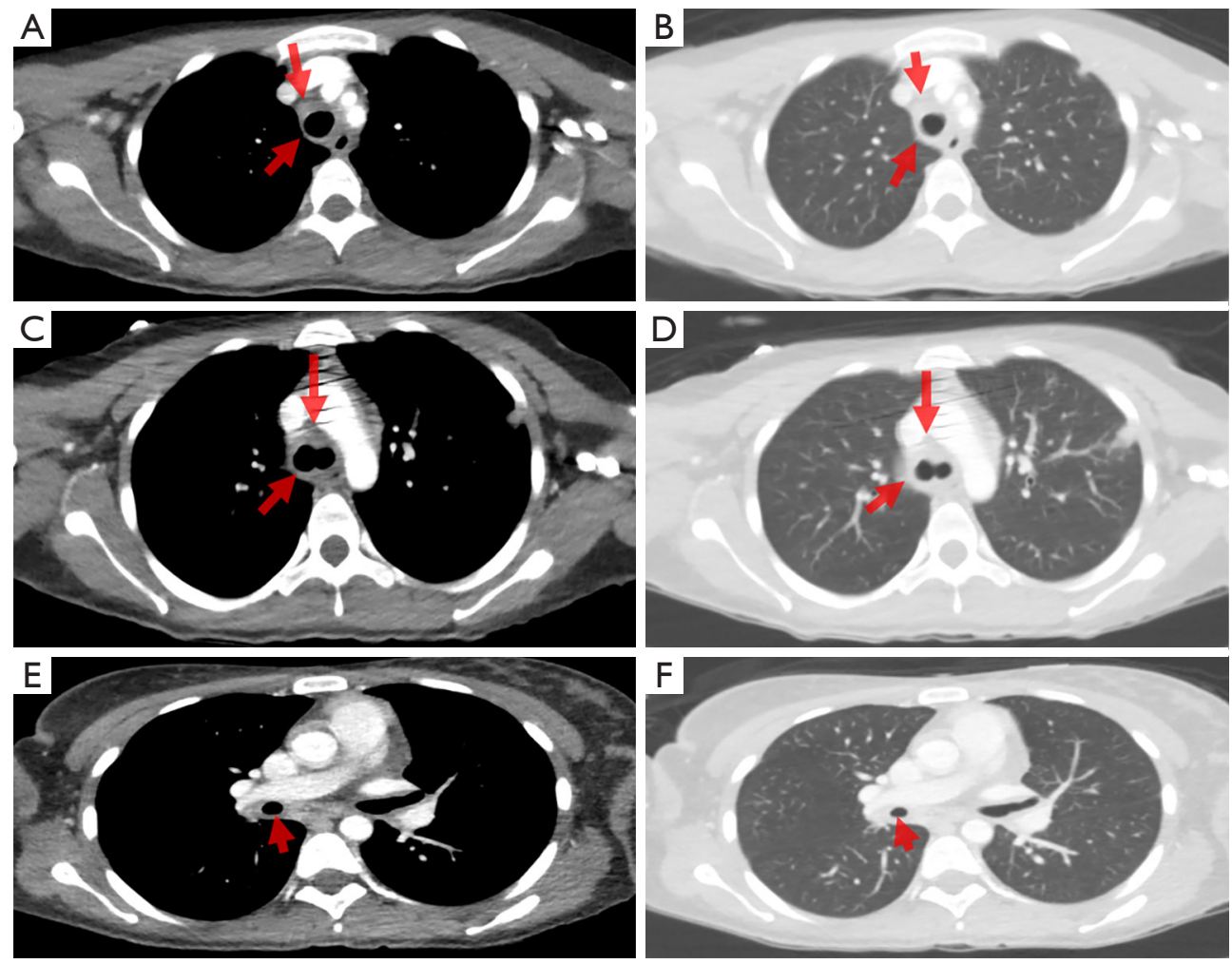

Figure 1 Contrast enhanced axial CT thorax in soft tissue (left) and lung (right) windows, demonstrates circumferential tracheal wall thickening above the level of the carina (A,B), circumferential tracheobronchial wall thickening at the level of the carina (C,D), as well as bronchial wall thickening below the level of the carina $(\mathrm{E}, \mathrm{F})$, as indicated by the red arrows.

significant only for biopsy-proven UC, diagnosed 4 years prior, with one episode of inflammatory arthritis in her hands and knees 2 years after her initial UC diagnosis. She was previously taking mesalamine ( $4.8 \mathrm{~g} /$ day) but had discontinued it on her own 6 months prior to admission due to lack of symptoms. She did not recall ever receiving any treatments other than mesalamine.

Labs on arrival to our hospital were notable for a white blood cell count of $28,000 \mathrm{k} / \mathrm{mm}^{3}$ (94\% neutrophils, $5 \%$ lymphocytes); hemoglobin $7.5 \mathrm{~g} / \mathrm{dL}$; platelet count of $1,163 \mathrm{k} / \mathrm{mm}^{3}$; procalcitonin $0.34 \mathrm{ng} / \mathrm{mL}$; erythrocyte sedimentation rate $96 \mathrm{~mm} / \mathrm{h}$; C-reactive protein $17 \mathrm{mg} / \mathrm{dL}$; antinuclear antibody titer 1:160; Anti-myeloperoxidase antibody was not reactive; Anti-proteinase-3 antibodies reactive. Her last UC flare was 3 months prior to this admission, however she still had intermittent diarrhea and was therefore tested for Clostridium difficile infection; she tested positive.

Flexible bronchoscopy was performed as part of the work-up for her initial stridor, and showed multiple papillomatous-like lesions throughout the trachea (Figure 2), lending to initial concern for tracheobronchial papillomatosis with parenchymal involvement. Tracheobronchial biopsies showed ulcerated mucosa with fibrinopurulent exudate and focal re-epithelialization without the detection of granuloma formation (Figure 3). Pathology was negative for papillomatosis, viral cytopathic changes, evidence of fungal organisms or malignancy. Workup for infectious etiologies including tuberculosis as well as other autoimmune pathology such as sarcoidosis or granulomatosis with polyangiitis, were negative.

Empiric treatment with high-dose corticosteroids led to marked improvement in her respiratory status. The dose of steroids was tapered and the patient remained symptomfree at discharge with plans to undergo repeat chest CT 4 weeks after initiation of steroids to monitor for resolution of lung nodules. Due to active Clostridium difficile infection, a colonoscopy was deferred. Biopsy and serologic findings, in the absence of an alternate etiology, support a diagnosis of UC-related lung disease. She was scheduled for 
outpatient pulmonary and GI appointments but failed to follow-up.

\section{Discussion}

IBD is well known to cause extraintestinal manifestations including uveitis, arthritis, erythema nodosum, pyoderma gangrenosum, sclerosing cholangitis, and hemolytic anemia $(6,7)$. Aside from the occasional case report, airwayassociated disease is rarely acknowledged as a complication of IBD. Lung involvement may be subclinical and incidentally found on PFTs or present as subglottic-glottic stenosis, tracheobronchitis, bronchiectasis, cryptogenic organizing pneumonia, or interstitial lung disease (3,8-10).

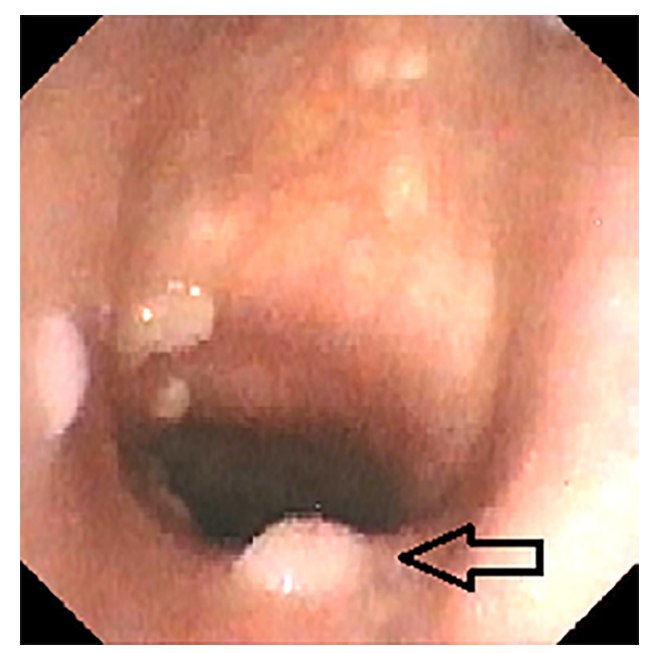

Figure 2 Multiple papillomatous-like lesions (arrow) and airway inflammation in the trachea, seen on flexible bronchoscopy.
A concrete link between lung disease and IBD is difficult to establish. Confounding factors include the fact that medications used for the treatment of IBD including sulfasalazine, mesalamine, methotrexate, and tumor necrosis factor-alpha inhibitors, are also known to cause airway disease. Thus, the association is typically made after other etiologies have been ruled out and based on histopathology findings from bronchoscopy. Also important to note is the fact that pulmonary manifestations may precede a diagnosis of IBD or occur during quiescent periods of the disease. Camus et al reported a retrospective review of 27 cases of UC-related pulmonary disease and found that $12 \%$ of those patients developed respiratory symptoms prior to the diagnosis of UC, and only $3 \%$ developed respiratory and gastro-intestinal symptoms concurrently (11).

Multiple studies have demonstrated that patients with IBD have abnormal pulmonary function tests (PFTs), as compared to healthy controls, even when they are clinically asymptomatic. Those with UC are more likely to have abnormal PFTs as compared to those with $\mathrm{CD}$, and restrictive lung findings are more common than obstructive. The most common abnormality found was a decrease in diffusion capacity of the lung for carbon monoxide (DLCO). Changes in forced expiratory volume in one second (FEV1) and the inspiratory vital capacity (IVC) were also noted but varied depending on disease activity (12).

A wide variety of bronchoscopic findings have been reported including severe tracheal narrowing, ulcerated mucosa and an ulcerated cobblestone appearance (1). Typical histopathological findings include abundant inflammatory cells within the epithelium without evidence of granuloma formation (13).
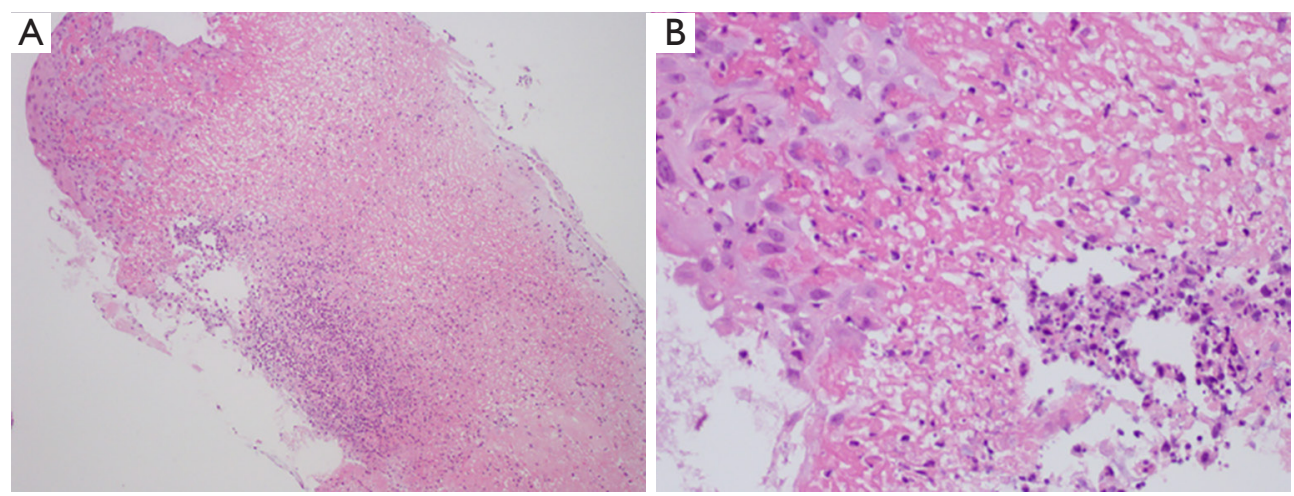

Figure 3 Pathology demonstrating ulcerated tracheal mucosa with fibrinopurulent exudate and focal re-epithelialization at 200× (A) and 400× (B) magnification (H\&E stain). 
Systemic steroids are the mainstay of treatment for suspected IBD-related airway disease and led to rapid improvement in most cases with large airway involvement $(3,11)$. Temporarily, a mixture of helium and oxygen (heliox) may be used in patients who present with airway obstruction symptoms due to tracheobronchitis (9). One case reported resolution of symptoms and improvement in tracheobronchial lesions on repeat bronchoscopy after treatment with inhaled corticosteroids alone (7). In more severe cases, the addition of cyclophosphamide to a steroid regimen was associated with improved outcomes (5). Treatment of the underlying IBD with mesalamine or other available disease controllers should be initiated simultaneously. In conclusion, tracheobronchitis is a rare extraintestinal manifestation of $\mathrm{UC}$ and may be life-threatening. Increased recognition amongst physicians is imperative as prompt diagnosis and aggressive management are the keys to a better outcome.

\section{Acknowledgements}

None.

\section{Footnote}

Conflicts of Interest: The authors have no conflicts of interest to declare.

Informed Consent: Written informed consent was obtained from the patient for publication of this manuscript and any accompanying images.

\section{References}

1. Hiyoshi M, Kawai K, Shibuya M, et al. Tracheobronchitis with dyspnea in a patient with ulcerative colitis. Intern Med 2015;54:749-53.

Cite this article as: Farkas ZC, Keshishyan S, Chakinala RC, Frager S, Saeed F, Yusuf Y, Shilagani C, Bodin R, Harris $\mathrm{K}$, Aronow WS. Tracheobronchitis with stridor in a patient with ulcerative colitis. Ann Transl Med 2018;6(22):445. doi: 10.21037/atm.2018.10.47
2. Veloso FT. Extraintestinal manifestations of inflammatory bowel disease: do they influence treatment and outcome? World J Gastroenterol 2011;17:2702-7.

3. Shad JA, Sharieff GQ. Tracheobronchitis as an initial presentation of ulcerative colitis. J Clin Gastroenterol 2001;33:161-3.

4. Kar S, Thomas SG. A case of tracheobronchitis in ulcerative colitis: a review of literature. Clin Respir J 2009;3:51-4.

5. Valletta E, Bertini M, Sette L, et al. Early bronchopulmonary involvement in Crohn disease: a case report. BMC Gastroenterol 2001;1:13.

6. Majewski S, Piotrowski W. Pulmonary manifestations of inflammatory bowel disease. Arch Med Sci 2015;11:1179-88.

7. Asami T, Koyama S, Watanabe $\mathrm{Y}$, et al. Tracheobronchitis in a patient with Crohn's disease. Intern Med 2009;48:1475-8.

8. Bayraktaroglu S, Basoglu O, Ceylan N, et al. A rare extraintestinal manifestation of ulcerative colitis: tracheobronchitis associated with ulcerative colitis. J Crohns Colitis 2010;4:679-82.

9. Janssen WJ, Bierig LN, Beuther DA, et al. Stridor in a 47-year-old man with inflammatory bowel disease. Chest 2006;129:1100-6.

10. Lu DG, Ji XQ, Liu X, et al. Pulmonary manifestations of Crohn's disease. World J Gastroenterol 2014;20:133-41.

11. Camus P, Piard F, Ashcroft T, et al. The lung in inflammatory bowel disease. Medicine (Baltimore) 1993;72:151-83.

12. D'Andrea N, Vigliarolo R, Sanguinetti CM. Respiratory involvement in inflammatory bowel diseases. Multidiscip Respir Med 2010;5:173-82.

13. Yamamoto AK, Babar JL. Case 184: ulcerative tracheobronchitis. Radiology 2012;264:609-13. 